\title{
Neural-network based approach to optimize THz computer generated holograms
}

\author{
Mateusz Surma, ${ }^{* 1}$ Mateusz Kaluza, ${ }^{1}$ Patrycja Czerwińska, ${ }^{1}$ Paweł Komorowski, ${ }^{2}$ and Agnieszka Siemion ${ }^{1}$ \\ ${ }^{1}$ Faculty of Physics, Warsaw University of Technology, Koszykowa 75, 00-662 Warsaw, Poland \\ ${ }^{2}$ Institute of Optoelectronics, Military University of Technology, Kaliskiego 2, 00-908 Warsaw, Poland
}

Received October 14, 2021; accepted December 20, 2021; published December 31, 2021

\begin{abstract}
Terahertz (THz) optics often encounters the problem of small f-number values (elements have relatively small diameters comparing to focal lengths). The need to redirect the $\mathrm{THz}$ beam out of the optical axis or form particular intensity distributions resulted in the application of iterative holographic methods to design $\mathrm{THz}$ diffractive elements. Elements working on-axis do not encounter significant improvement while using iterative holographic methods; however, for more complicated distributions the difference becomes meaningful. Here, we propose a totally different approach to design $\mathrm{THz}$ holograms, utilizing a neural network based algorithm, suitable also for complicated distributions.
\end{abstract}

Fast developing terahertz (THz) technology finds applications in many fields of science and industry such as optics, medicine, security, or non-destructive testing [1-3]. Each new application requires specific optical elements that will allow for full use of the potential of $\mathrm{THz}$ radiation under given conditions. Thus, new solutions allowing to meet market needs are still requested. Nowadays, thin diffractive optical elements (DOEs) are used instead of thick refractive lenses. The continuous phase changes, kinoform coding method, allow to redirect the whole electromagnetic radiation into a single diffraction order, which significantly increases the efficiency of the THz diffractive structures [4-5].

More advanced DOEs can be designed as synthetic holograms. Synthetic holography is based on calculating complex transmittance that, when illuminated, redirects the radiation to form the image of an object Therefore, designed wavefront shapes can be more advanced and complex compared to classic holography [5] or designing DOEs using analytical equations.

Regrading applicational requirements, synthetic holography mostly utilizes different kinds of iterative methods like the Gerchberg-Saxton, also called the Iterative Fourier transform Algorithm (IFTA), or the ping-pong algorithms [6-7]. Such algorithms perform a number of iterations propagating the wavefront between the hologram and the image planes. In each plane a particular amplitude distribution is forced, while the phase is being optimized. Thus, the generated phase distribution in the hologram plane results in forming the

${ }^{*}$ E-mail: mateusz.surma.dokt@pw.edu.pl desired, previously defined, amplitude distribution in the image plane.

Nowadays, the additive manufacturing technique is more and more often used to create the designed $\mathrm{THz}$ DOEs. Such techniques use polymer materials in the form of powder, wire (filament), or resin to deposit material layer by layer. In the case of lower $\mathrm{THz}$ frequencies additive techniques guarantee a sufficiently high resolution of the manufacturing process [8]. Such techniques allow both for fast prototyping and good optical properties of fabricated components due to the high performance of polymer materials in the $\mathrm{THz}$ radiation range [9].

This work compares the $\mathrm{THz}$ phase kinoform lens with a synthetic hologram structure designed with iterative techniques. Moreover, we present a synthetic hologram generated by a neural network (NN). The network has been trained by the backpropagation method with stochastic gradient descent using an adaptive learning rate (ADAM). It is an innovative solution in the field of designing synthetic holograms. A detailed description of the algorithms, as well as the source code can be found in the Wolfram Community blog post [10]. Th first approach to optimize DOEs with this method was described only using numerical simulations in [11]. The performance of the NN structure was simulated numerically. Then, a three-dimensional model of the hologram was created and fabricated using the additive manufacturing technique.

Three different structures were designed using different approaches to obtain DOEs with the design wavelength $\lambda=2.141 \mathrm{~mm}$ (frequency $140 \mathrm{GHz}$ ), the diameter $D=100$ $\mathrm{mm}$ and focal length $f=700 \mathrm{~mm}$. The first method calculated the phase distribution $(\varphi)$ according to the analytical non-paraxial converging lens formula:

$$
\varphi(x, y)=-\frac{\lambda}{2 \pi} \sqrt{x^{2}+y^{2}+f^{2}},
$$

where $\lambda$ is the design wavelength, $f$ is the focal length, $x$ and $y$ are the cartesian coordinates.

The second approach was to design a structure using the iterative method based on the ping-pong algorithm. The single iteration process consisted of forward propagation from the hologram plane to the image plane, where the desired amplitude distribution - the focal spot 
diameter - was forced. Then, backward propagation from the image plane to the hologram plane was performed. The process was repeated 10 times and the desired intensity patterns in both planes were applied. All propagations were realized using the modified convolution method [12]. The iterated hologram was also calculated for different forced amplitudes with smaller diameters of the desired focal spots to investigate the influence of the focal spot size on the phase distribution patterns.

In the third approach, a structure realizing the same diffractive task - on-axis radiation focusing - was designed by means of neural network training.

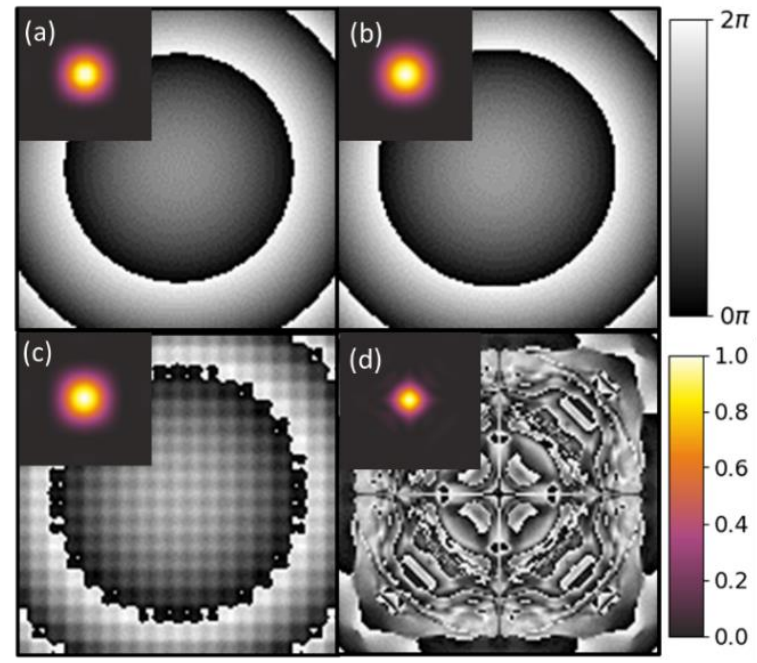

Fig. 1. The comparison of the converging lens created by: a) an analytical phase kinoform equation (Airy disc size $-36.6 \mathrm{~mm}$ ) and iterative synthetic holograms with different predefined focal spot diameter sizes b) $d=18 \mathrm{~mm}$, c) $d=9 \mathrm{~mm}$ and d) synthetic hologram calculated by NN (predefined $18 \mathrm{~mm}$ ). Theoretical intensity

distributions are presented in the left top corners of images; they have been normalized according to the scale presented in the right bottom, while the phase distributions are described by the greyscale palette presented in the top right.

In all simulations a grayscale $128 \times 128$ px bitmap has been generated. Each pixel contained a value from 0 to 255 that corresponded to a phase retardation from 0 to $2 \pi$.

The phase distributions, corresponding to the theoretical equation (a) with an Airy disc size equal to $36.6 \mathrm{~mm}$, the iterative algorithm with a forced focal spot diameter of $18 \mathrm{~mm}$ (b) and $9 \mathrm{~mm}$ (c) as well as neural network based design with the forced focal spot equal to $18 \mathrm{~mm}$ (d) are presented in Fig. 1. Additionally, the simulated theoretical intensities in the focal planes $(f=$ $700 \mathrm{~mm}$ ) are shown in the insets. As it can be seen in the top row, the difference between the phase maps generated by the theoretical equation and iterative algorithm is unperceivable for the human eye. The difference in Fig. 1c is, however, much more likely to be noticed. The obtained phase map is more grainy and noisy, which is caused by reducing the size of the target focal spot used in the iteration process significantly below the diffraction limit. On the other hand, the obtained focal spot remained unchanged (its diameter cannot be reduced below the beforementioned diffraction limit), even though the forced focal spot was smaller than the Airy disc size. Since the phase distribution complexity and granularity have direct influence on the quality of manufacturing, the size of the forced focal spots should be carefully considered. The neural network based designed hologram phase distribution has a completely dissimilar and individual look that does not remind any of the previous ones in Fig. 1. Theoretically, the intensity of the focal spot simulated by NN approach creates a hologram that forms an image having a smaller diameter than the analytically designed one and introduces only a small background interference pattern around it.

The height of each pixel, corresponding to the bitmap's phase distribution $\varphi$, can be calculated according to the following equation (2):

$$
h(x, y)=\frac{\varphi(x, y) \lambda}{2 \pi(n-1)}
$$

where $n$ is the refractive index of the material and $\varphi$ is the phase distribution expressed in the cartesian coordinates.

The height map must be transformed into a real structure by creating a proper $3 \mathrm{D}$ file and a manufacturing method. To assure proper manufacturing quality a sampling of $900 \mu \mathrm{m}$ was chosen. Therefore, the 3D model of the synthetic hologram was created with a total horizontal size of $115.2 \times 115.2 \mathrm{~mm}+5 \mathrm{~mm}$ frame and a maximal height of $4.17 \mathrm{~mm}+0.5 \mathrm{~mm}$ substrate. Two different designing techniques of 3D models were analyzed. The first approach (simplest) is based on extruding each point (node) represented by pixel into the designed height level. Next, the nodes are connected forming triangles in the 3D space (for .stl file format). Thus, the obtained model representing the bitmap interpolates values between nodes. This solution can be applied to oversampled models; however, for small matrices such an approach introduces large errors. This method was used to manufacture a converging lens defined by the theoretical equation, where the phase distribution is smooth enough to assure proper quality of the DOE. The second method was based on building 3D blocks representing each pixel (each composed of 12 combined triangles) and extracting blocks to a calculated height level. This second method is crucial in extending relatively small matrix size bitmaps, guaranteeing a significantly better representation of the bitmap in the case of NN synthetic holograms according to the carried out simulations. Images obtained for NN 3D models with both methods are shown respectively in Fig. $2 \mathrm{a}$ and $2 \mathrm{~b}$.

The NN synthetic hologram was fabricated using the additive manufacturing technique - FDM (Fused Deposition Modelling) with the filament Cyclic Olefin Copolymer (COC), characterized by high stiffness, low deformation and extremely good optical properties. Its 
absorption coefficient is very low in the whole $\mathrm{THz}$ spectral range (for $140 \mathrm{GHz}$ it is equal to $0.05 \mathrm{~cm}^{-1}$ ), and its refractive index was equal to 1.513 . These properties guarantee extraordinary possibilities in the fabrication of phase THz DOEs. The horizontal and vertical resolutions of the 3D printer were equal to $450 \mu \mathrm{m}$ and $100 \mu \mathrm{m}$, respectively. It allowed to precisely represent each pixel of the bitmap in the manufacturing process. An analytically designed kinoform lens was produced in the same way.

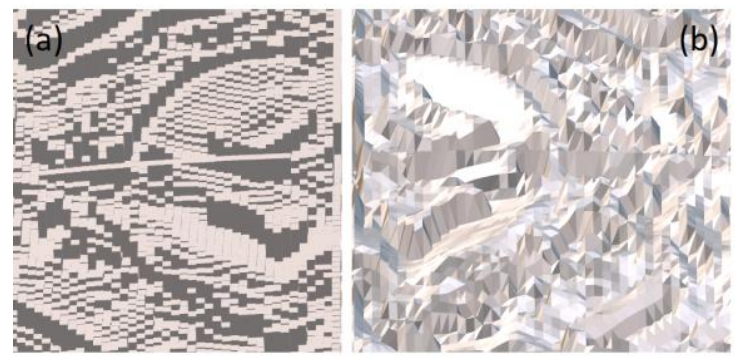

Fig. 2. The 3D models of the NN synthetic hologram rendered by two different methods: a) extending each bitmap's pixel separately,

b) interpolation of nodes with respect to pixels' height levels. Significant differences between models can be noticed.

Both the NN and the analytically designed kinoform lens were verified in the experimental setup. It consisted of a frequency multiplier (WR-5.1 from Virginia Diodes) emiting radiation at $140 \mathrm{GHz}$, parabolic mirror forming a quasi-plane wave, as well as a tested lens and a detector (Schottky diode with a hemispherical silicon lens). The precise position of the focal plane was experimentally adjusted for the minimal diameter and maximal intensity of the focal spots and slightly differed from the designed focal length, due to the imperfections of the incident wavefront. Raster scans of the intensity distributions were registered in XY plane (perpendicular to the optical axis) and are presented in Fig. 3.

Both structures allowed to obtain sharp focal spots in the desired positions. A classic off-axis diffractive lens generated from the analytical equation forms a nice round focal spot within the diffraction limit. The structure based on neural network design gives also a small and well focused spot. The background is larger than for classic DOE, but the intensity distributions were normalized, so taking into account the fact that the NN based structure had a smaller maximal intensity in the focal spot, it could result in larger background noise. Nevertheless, an extremely non-intuitive and non-predictable phase distribution can successfully focus the radiation into a focal spot slightly larger (by $0.6 \mathrm{~mm}$ according to their FWHMs, equal to $13.6 \mathrm{~mm}$ and $14.2 \mathrm{~mm}$, which corresponds to the $32.2 \mathrm{~mm}$ and $33.7 \mathrm{~mm}$ Airy disc size, respectively) unlike in the case of a classic DOE lens.

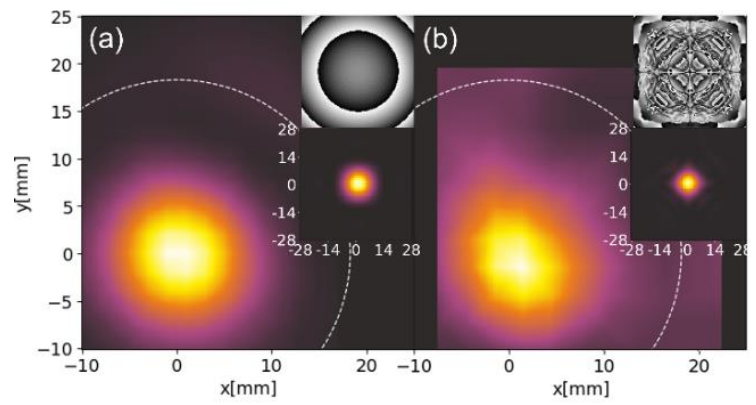

Fig. 3. The experimental results. The registered intensity (normalized) distributions for: (a) the lens designed according to the analytical equation, corresponding to Fig. 1a and (b) the NN-based algorithm, corresponding to Fig 1d. The white circles denote the size of an Airy disk for the designed structures $(\lambda=2.141 \mathrm{~mm}, f=700 \mathrm{~mm}, D=100$ $\mathrm{mm}$, circular aperture). In the insets - the structures phase maps and intensity distributions in the focal plane are shown for both cases.

Concluding, the converging lens designed by the NN algorithms was successfully developed and experimentally verified. The proposed converging lens has a totally different phase distribution, which is abstract and non-intuitive, especially comparing to the converging kinoform lenses and iterated holograms. The experimental results of both analytical equation based and NN based structures have shown that the proposed NN holographic lens is generating the focal spot as good as a classical lens. The larger focal spot size for the NN structure can result from scattering and shadow effect due to the fact that details of the phase map are much smaller than its height. However, this novel approach is opening new possibilities in the designing process of diffractive optical elements for $\mathrm{THz}$ radiation. From the manufacturing point of view it is crucial to adapt a $3 \mathrm{D}$ model to the complexity of phase transmittance to ensure a faithful representation of the phase map, which was realized by extending each pixel in the bitmap separately.

The research was funded by the National Science Centre, Poland under the OPUS-18 programme (2019/35/B/ST7/03909).

\section{References}

[1] Y. Tao, A. Fitzgerald, V. Wallace, Sensors 20(3), 712 (2020)

[2] J. O'Hara, S. Ekin, W. Choi, I. Song, Technologies 7(2), 43 (2019).

[3] L. Yu et al., RSC Advances 9(17), 9354 (2019).

[4] A. Siemion, Sensors 21(1), 100 (2020).

[5] A. Siemion, J. Infrared Millim. Terahertz Waves, 40(5), 477 (2019).

[6] M. Surma, I. Ducin, P. Zagrajek, A. Siemion, Appl. Sci. 9(4), 659 (2019).

[7] S. Banerji, B.Sensale-Rodriguez, Scientific Reports 9(1), 5801 (2019).

[8] J. Sun, F. Hu, Int. J RF Microw Comput Aided Eng. 30(1) (2020).

[9] E. Castro-Camus, M. Koch, A.I. Hernandez-Serrano, J. Appl. Phys. 127(21), 210901 (2020)

[10] https://community.wolfram.com/groups/$/ \mathrm{m} / \mathrm{t} / 2028026 ? \mathrm{p} \% 20479 \% 20 \mathrm{p}$ auth=blBtLb5d

[11] P. Komorowski et al., Opt. Expr. 29(7), 11243 (2021).

[12] M. Sypek, Opt. Comm. 116(1-3), 43 (1995). 